\title{
Cervical Cancer Screening with Liquid Cytology in Women with Developmental Disabilities
}

\author{
Shahryar K. Kavoussi, M.D., M.P.H., Yolanda R. Smith, M.D., M.S., \\ Susan D. Ernst, M.D., and Elisabeth H. Quint, M.D.
}

\begin{abstract}
Objective: To evaluate the use of liquid cytology in Pap smears in women with developmental disabilities (DD) for endocervical cell yield and abnormalities, via speculum examination or blind technique.

Methods: We used retrospective chart review of gynecological visits by women with DD from October 2002 to November 2005. Cervical cytology screening included speculum examination or blind technique. Endocervical cell yield was analyzed via Pearson's chi-square test.

Results: Of 240 attempted liquid cytology Pap smears, 199 (82.9\%) were completed. Of these, 193 met inclusion criteria for the study, and $120(62.2 \%)$ contained endocervical cells. The endocervical cell yield with liquid cytology/speculum was $80.0 \%$ and was $43.6 \%$ with liquid cytology/blind $(p<0.001)$. Two blind smears $(1.0 \%)$ were abnormal; both revealed atypical squamous cells of undetermined significance (ASCUS) with subsequent negative human papillomavirus (HPV) typing.

Conclusions: Cervical screening with liquid cytology in women with DD provides an overall rate of endocervical cells of approximately $44 \%-80 \%$ depending on the technique used. Although this is much lower than in the general population, this compares favorably with slide Pap smear in women with DD. The $44 \%$ yield of endocervical cells and the finding of abnormal Pap smears with the blind technique suggest this is a reasonable alternative for obtaining Pap smears in women with difficult pelvic examinations who otherwise would not receive cervical screening.
\end{abstract}

\section{Introduction}

G YNECOLOGICAL CARE IN WOMEN with developmental disabilities (DD) poses numerous challenges for the caregiver as well as the patient. Obtaining an adequate menstrual and sexual history is often difficult because of communication issues and lack of available historical data. Therefore, the occurrence of past sexual activity may remain unclear in this patient population. A pelvic examination, including a Papanicolaou (Pap) smear, is often especially difficult due to patient cooperation and physical handicaps. ${ }^{1}$

The Pap smear is an important tool for cervical screening and has significantly reduced the incidence of and death from cervical malignancies. ${ }^{2}$ Technology for cervical cytology has greatly changed over the last decade, from the conventional Slide Pap smear to the now commonly used liquid-based cytology. The presence or absence of endocervical cells determines the adequate sampling of the transformation zone and is, therefore, used in studies as an indicator of successful sampling. ${ }^{3}$ Previous studies with slide Pap smear showed that the cytobrush was more effective in the collection of endocervical cells $(89 \%-99 \%$ yield) than was the cotton swab $(62 \%-87 \%){ }^{4-6}$ Liquid-based cytology has been found to be as efficacious as, ${ }^{7}$ or superior to, ${ }^{8,9}$ slide Pap smear for the diagnosis of cervical intraepithelial neoplasia II-III (CIN II-III) and for decreasing the number of inadequate specimens.

There have been very few studies in the past addressing cervical cytology in women in whom pelvic examinations are difficult. ${ }^{10}$ All traditional techniques have been found to be suboptimal, including a described nonspeculum blind swab technique with a cotton tip, which had a very low yield of endocervical cells but was often the only option in women with a difficult pelvic examination. ${ }^{1,10}$

The primary aim of this study was to evaluate the use of liquid cytology for cervical screening in women with DD. We assessed the yield of endocervical cells and abnormal Pap smears using liquid cytology Pap smears and compared examination techniques (use of speculum vs. a blind swab technique). 


\section{Materials and Methods}

A retrospective electronic chart review was performed of clinic visits by women who attended a reproductive health clinic that provides care exclusively for women with DD at the University of Michigan from October 2002 to November 2005. All Pap smears were performed by an obstetrician-gynecologist, and no intravenous or oral sedation was administered. In October 2002, Pap smear technology was converted to liquid cytology (ThinPrep, Cytyc Corporations, Marlborough, MA). The study included all women $<66$ years of age who underwent an attempted Pap smear with liquid cytology at the clinic. Women with a prior hysterectomy were excluded. The electronic charts were reviewed by a person who was not involved in the gynecological care of these patients. In reviewing each patient visit, a standardized clinic visit form was created that included the patient's age, level of developmental delay, description of the pelvic examination, presence of an attempted Pap smear, and use and outcomes of liquid-based cytology.

The level of developmental delay was noted as it was described in the chart in the gynecology or other medical notes (mild, moderate, severe, unable to determine from the chart). The pelvic examination included use of a speculum or a blind technique. The blind technique was used only in those women who could not tolerate a speculum examination. This technique consists of the advancement of one finger in the vagina, palpating the cervical os, and sliding a cytobrush over the finger to guide it to the cervical os in an effort to obtain endocervical cells. If possible a spatula was also used; however, this was rarely tolerated. All cervical cytology results were examined for the presence or absence of endocervical cells, as well as cytological abnormalities.

Statistical analyses were performed via Pearson's chisquare tests on completed cervical cytology screening examinations. Although some patients had multiple revisits and repeat examinations, each attempted Pap smear was weighted equally, and the percentages of Pap smears that contained endocervical cells were calculated. Confidence intervals (CI) were not obtained, as not all the examinations were statistically independent. Approval was obtained from the Institutional Review Board of the University of Michigan prior to conducting the study.

\section{Results}

From October 2002 to November 2005, there were 386 clinic visits by 186 women with DD who met study age criteria, and 240 visits included a Pap smear or an attempted Pap smear. The average age of the patients at the time of Pap smear was 41.0 years, with a range of 17-65 years. In the 186 patients, the level of developmental delay was described as severe in $67(37.1 \%)$, moderate in $10(5.4 \%)$, mild in 16 $(8.6 \%)$, and unreported in 91 (48.9\%).

Of the 240 Pap smears attempted, 199 (82.9\%) were able to be obtained, and $194(80.8 \%)$ met criteria. One blind-obtained specimen was acellular and was excluded from the analysis; therefore, 193 Pap smears were studied. Of these, $120(62.2 \%)$ contained endocervical cells. For a comparison of those patients in whom a speculum could be used vs. those in whom that was not possible (blind technique), the 193 Pap smears were compared by technique: use of a speculum $(n=$ $99)$ vs. the blind technique $(n=94)$. This comparison dem- onstrated that with liquid cytology, $79(80.0 \%)$ of smears contained endocervical cells when a speculum was used, and 41 $(43.6 \%)$ contained endocervical cells when a blind technique was used (chi-square $=26.84, p<0.001$ ) (Fig. 1). Two patients had abnormal Pap smears; both were classified as atypical squamous cells of undetermined significance (ASCUS), with subsequent negative human papillomavirus (HPV) typing. Review of these cases shows that 1 patient had a normal follow-up, and 1 patient did not return for follow-up.

\section{Discussion}

Although there is difficulty in performing Pap smears for women with DD, obtaining cervical cells for cancer screening was feasible in the majority of subjects (82.9\%). The yield of endocervical cells with liquid cytology ranged from $43.6 \%$ with the blind technique to $80 \%$ for those who were able to tolerate a speculum. Although these numbers are lower in than the general population, they are statistically higher than previously published rates with slide Pap smear techniques. ${ }^{10}$ In this previous study, ${ }^{10}$ the slide Pap smear demonstrated an overall yield of endocervical cells of $34 \%$, whereas the use of a speculum and the blind technique provided endocervical cells in $58 \%$ and $18 \%$ of Pap smears, respectively.

The presence of endocervical cells has been used as a measure of quality or adequate sampling of the transformation zone. There is controversy as to whether an endocervical cell component is necessary to increase detection of cervical neoplasia, but it is still included in the Bethesda system to describe specimen adequacy. ${ }^{11,12}$ Therefore, we chose the presence of endocervical cells as our measure of adequate Pap smear screen for this study. We find acceptable values of endocervical cells, improved over previous techniques, in our population. These values are lower than in the general population, even with a speculum, because of the difficult examinations as a result of challenges with patient cooperation, communication, and physical disabilities. From these observations, we conclude that if a speculum cannot be used, the blind technique with liquid cytology, although neither ideal nor a replacement for the standard technique, may yield endocervical cells and may identify abnormalities.

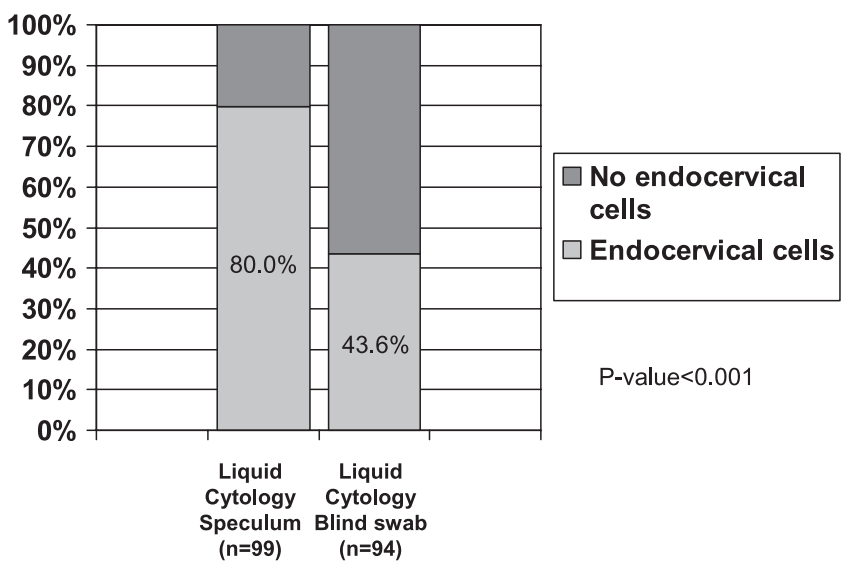

FIG. 1. Comparison of endocervical cell yield with liquid cytology, speculum vs. blind swab technique. 
Examinations in women with DD are often helped by special techniques. This may include a two-visit approach, with the first visit to help the patient become comfortable with the provider and the second for the actual examination. A trusted caregiver present may help the patient relax. Stirrups are seldom used, and a variety of positions may facilitate the examinations, including the frog leg position, the side position, or any position that does not abduct the legs significantly. A long narrow Huffman speculum (adolescent speculum) is advocated as opposed to a pediatric speculum, which is too short for the adult vagina. Lidocaine gel on the introitus has also been advocated by some. ${ }^{13}$ In the case of an abnormal blind Pap smear, an examination under anesthesia for colposcopy or treatment may be warranted.

Abnormal cytology findings accounted for $1.0 \%$ of Pap smears in the present study and for $0.3 \%$ in a previous study in this population. ${ }^{10}$ These percentages compare to an abnormal conventional Pap smear rate of $1.5 \%-21.9 \% 14$ and an abnormal liquid cytology Pap smear rate of $21 \%-22 \%$ in the general gynecological population. ${ }^{15}$ The presence of abnormal cytology in women with DD is below the national prevalence, which has been reported as $2.3 \%$ in a study of 1,045,059 conventional samples. ${ }^{16}$ We suspect that most of our patients are not often engaged in sexual activities, but it is difficult to obtain a complete history because of communication issues and record keeping of current and past sexual activity. Unfortunately, the potential for being victims of sexual abuse exists among these patients, thereby increasing the risk of HPV infection. The actual incidence of abuse is difficult to gauge; however, it has been reported as $13 \%-63 \%$ in previous studies. ${ }^{17-20}$

This study has several limitations. The retrospective nature of this study is not ideal and may lead to bias; however, the ability to perform a prospective study in this population is limited because of difficulties with consent, continuity of care, and follow-up. Another issue may be the age of our patients. As 13 of the women in our study were over the age of 55 years, the postmenopausal regression of columnar epithelium upward into the endocervical canal may account for the lack of endocervical cells in some samples.

When such issues as patient tolerance of examinations and the infrequency of high-grade intraepithelial lesions are considered, the question arises as to whether strict adherence to cervical cytology screening recommendations is necessary in women with developmental delay or whether special guidelines could be established. Currently, the recommendations for cervical cytology screening among women in the general population are applied to this specific patient population. The American College of Obstetricians and Gynecologists (ACOG) and the American Cancer Society (ACS) recommend that screening should begin either 3 years after the initiation of intercourse or at age 21, depending on which event occurs first. ${ }^{3,21}$ ACOG and ACS recommendations include annual cervical cytology screening before the age of 30 and extension of the screening interval to every $2-3$ years in women aged $\geq 30$ years provided they have had three consecutive negative Pap smears and no history of CIN II or III, diethylstilbestrol exposure, or an immunocompromised status, including infection with HIV. ${ }^{3,21}$ The combination of negative cytology and negative HPV DNA testing has been reported to yield negative predictive values of $99 \%-100 \% .^{22}$ Screening every 3 years in women with such results could be beneficial in a population such as those with DD. Whether blind screening with HPV only will be helpful for this group of women will need to be researched.

Clearly, the data currently available are not of sufficient size to make predictions as to the optimal time interval for cervical cancer screening in women with DD. However, the relatively low number of abnormal Pap smears and the minimal level of pathology ${ }^{23}$ support not undertaking aggressive screening, which may include anesthesia or sedation, for this patient population for preventive healthcare. Future studies need to address optimal screening intervals, the use of HPV testing in women with DD, and the use of the HPV vaccine.

\section{Conclusions}

Cervical screening with liquid cytology in women with DD gives an overall rate of endocervical cells of approximately $44 \%-80 \%$ depending on the technique used. Although this is much lower than in the general population, this compares favorably with slide Pap smears in women with DD. With a $44 \%$ yield of endocervical cells, although less than ideal, the blind technique is a reasonable alternative for obtaining Pap smears in women with difficult pelvic examinations who may otherwise get no cervical cancer screening.

\section{Acknowledgments}

This work was supported by the University of Michigan Department of Obstetrics and Gynecology.

\section{Disclosure Statement}

The authors have no conflicts of interest to report.

\section{References}

1. Elkins TE, McNeeley SG, Rosen D, et al. Clinical observation of a program to accomplish pelvic exams in difficult to manage patients with mental retardation. Adolesc Pediatr Gynecol 1988;1:195-198.

2. Reies LAG, Kosary CL, Hankey BF, et al. SEER cancer statistics review, 1973-1996. Bethesda, MD: National Cancer Institute, 1999.

3. Cervical cytology screening. ACOG Practice Bulletin No. 45. American College of Obstetricians and Gynecologists. Obstet Gynecol 2003;102:417-427.

4. Kristensen GB, Holund B, Ginsted P. Efficacy of the cytobrush versus the cotton swab in the collection of endocervical cells. Acta Cytol 1989;33:849-851.

5. Patrissi GA, Lee D. Cytobrush was superior to the standard cotton swab and spatula in sampling the endocervical canal. J Reprod Med 1992;37:72.

6. Dehbashi S, Honarvar M, Montazer NR. Comparison of cotton swab-spatula and cytobrush-spatula for cervical cytology. Int J Gynaecol Obstet 2002;76:185-187.

7. da Gloria Mattosinho de Castro Ferraz M, Nicolau SM, Stavale JN, et al. Cervical biopsy-based comparison of a new liquid-based thin-layer preparation with conventional pap smears. Diagn Cytopathol 2004;30:220-226.

8. Biscotti CV, O'Brien DL, Gero MA, et al. Thin-layer Pap test vs. conventional Pap smear. Analysis of 400 split samples. J Reprod Med 2002;47:9-13. 
9. Cox JT. Liquid-based cytology: Evaluation of effectiveness, cost-effectiveness, and application to present practice. J Natl Compr Canc Netw 2004;2:597-611.

10. Quint EH, Elkins TE. Cervical cytology in women with mental retardation. Obstet Gynecol 1997;89:123-126.

11. Apgar BS, Zoschnick L, Wright TC. The 2001 Bethesda system terminology. Am Fam Physician 2003;68:1993-1998.

12. Selvaggi SM, Guidos BJ. Endocervical component: Is it a determinant of specimen adequacy? Diagn Cytopathol 2002; 26:53-55.

13. Quint EH. Menstrual issues in women with developmental disabilities. In: Menstrual disorders. American College of Physicians' Women's Health Series, ACP, 2006: Chapter 8.

14. Bernstein SJ, Sanchez-Ramos L, Ndubisi B. Liquid-based cervical cytologic smear study and conventional Papanicolaou smears: A meta-analysis of prospective studies comparing cytologic diagnosis and sample adequacy. Am J Obstet Gynecol 2001;185:308-317.

15. Abulafia O, Pezzullo JC, Sherer DM. Performance of ThinPrep liquid-based cervical cytology in comparison with conventionally prepared Papanicolaou smears: A quantitative survey. Gynecol Oncol 2003;90:137-144.

16. Sadeghi SB, Sadeghi A, Robboy SJ. Prevalence of dysplasia and cancer of the cervix in a nationwide, planned parenthood population. Cancer 1988;61:2359-2361.

17. Chamberlain A, Rauh J, Passer A, et al. Issues in fertility control for mentally retarded female adolescents: Sexual activity, sexual abuse, and contraception. Pediatrics 1984;73:445-450.

18. Elvik SL, Berkowitz CD, Nicholas E, et al. Sexual abuse in the developmentally disabled: Dilemmas of diagnosis. Child Abuse Negl 1990;14:497-502.
19. Young ME, Nosek MA, Howland C, et al. Prevalence of abuse of women with physical disabilities. Arch Phys Med Rehabil 1997;78:S34-38.

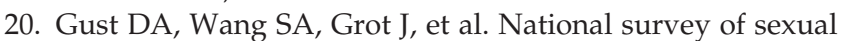
behavior and sexual behavior policies in facilities for individuals with mental retardation/developmental disabilities Ment Retard 2003;41:365-373.

21. Saslow D, Runowicz CD, Solomon D, et al. American Cancer Society guidelines for the early detection of cervical neoplasia and cancer. CA Cancer J Clin 2002;52:342-362.

22. Human papillomavirus. ACOG Practice Bulletin No. 61. American College of Obstetricians and Gynecologists. Obstet Gynecol 2005;105:905-918.

23. Jaffe JS, Timell AM, Eisenberg MS, et al. Low prevalence of abnormal cervical cytology in an institutionalized population with intellectual disability. J Intellect Disabil Res 2002; 46:569-574.

\author{
Address reprint requests to: \\ Elisabeth H. Quint, M.D. \\ Department of Obstetrics and Gynecology \\ University of Michigan Health Systems \\ 1500 E. Medical Center Drive, Room L4100 \\ Women's Hospital \\ Ann Arbor, MI 48109-0276
}

E-mail: equint@med.umich.edu 